\title{
Enhanced Timing Advance In TDM Cellular Radio Systems
}

\author{
R. Whitlock, T. Brown
}

The rationale for this letter is to present a method which would enhance the resolution and accuracy of Timing Advance (TA) in 2G GSM TDMA and 3G UMTS TDD networks. The method would enable a stationary or slow moving Mobile Station (MS) to determine its location using trilateration, with enhanced resolution and accuracy. An outstanding feature of the method is that the only change required is to software.

Low resolution TA samples would be processed into enhanced resolution measurements by applying fractional bit perturbations (dither) over an extended sampled measurement period. The existing TA mechanism and word length for ensuring correct MS uplink arrival times would remain unchanged. This letter proposes that Enhanced Timing Advance (ETA) could provide a low risk, low cost alternative to location finding technologies such as Angle of Arrival (AOA), Time of Arrival (TOA), Enhanced Observed Time Difference (E-OTD), Uplink Time Difference of Arrival (U-TDOA) and GPS, and at the same time satisfy the FCC mobile E-911 accuracy requirement.

Introduction: Timing Advance (TA) is a technique [1] used to ensure that signals originating from a MS arrive at the Base Station (BS) at the correct time within the allocated time slot. This is essential in TDMA systems such as GSM where the distance between the MS and the BS could be as little as a few metres, or as far as 
$35 \mathrm{~km}$. This also applies to UMTS TDD macro-cells, where propagation times need to be taken into account.

This letter describes a method that would improve the resolution and accuracy of currently implemented TA techniques. The method should enable MSs to calculate more accurately their range to cellular BSs to achieve a more accurate positional fix. The method requires the application of unbiased timing perturbations between transmission of the downlink signal and processing of the received uplink signal of TA measurements. This could be achieved by applying the timing perturbations to the downlink transmission timing, or to the uplink measurement timing window.

\section{Limitations of the TA Method:}

The TA method, as implemented by GSM systems, uses a coarse granularity measurement and signal representation. It is the uplink transmission advance (delayed by 3 frames) represented as a 6 bit number in units of $3.69 \mu \mathrm{S}$. This time step corresponds to a round trip propagation distance of approximately $1100 \mathrm{~m}$, providing a range resolution of $550 \mathrm{~m}$.

This letter presents a method that should reduce the granularity of the TA measurement, which together with reducing the MS timebase error, should provide a means, using trilateration [2], with which to increase location finding resolution and accuracy. This would enable Timing Advance to compare favorably with existing high resolution technologies in satisfying the FCC mobile E-911 accuracy requirements which specify 100 metres for $67 \%$ of calls and 300 metres for $95 \%$ of calls [3].

\section{Enhanced TA (ETA) Method:}

The achievable accuracy of this method would be limited by timing synchronisation errors between the MS and BS, which therefore also need to be addressed. 
Timebase synchronisation may be achieved by various methods. 3GPP specify that synchronisation uses the Synchronisation Channel $(\mathrm{SCH})$ burst which has been specifically designed for the purpose. Following initial synchronisation, 3GPP specifies that adjustments should take place at intervals of not less than 1 second and not greater than 2 seconds, until the timing difference is less than $1 / 2$ symbol period. If the timing difference between MS and BS exceeds $\pm 2 \mu \mathrm{S}$, it shall be reduced in $1 / 4$ symbol steps to less than $1 / 2$ symbol period [4].

Unfortunately, because of this non-linear timebase control mechanism, unnecessarily bias would result in the measurement set. In addition, the timebase update rate of 1 to 2 seconds is too slow.

Providing a means to calculate and report enhanced resolution measurements would require improved timebase accuracy beyond that achievable using the 3GPP synchronisation algorithm. One way that this may be achieved, is to use the frequent $\mathrm{SCH}$ bursts (which occur every 10 frames or $46.15 \mathrm{~ms}$ ). Instead of imposing a $\pm 2 \mu \mathrm{S}$ error limit before taking corrective action every 1 to 2 seconds as is presently the case, the proposed strategy is to act on each burst, advancing or delaying the timebase by a quarter of a symbol, depending on whether $\mathrm{SCH}$ correlation shows the timebase to be lagging or leading the BS respectively. This will result in the MS timebase hunting about the BS timebase within a maximum error of $\pm 1 / 4$ of a symbol during the measurement set period, without hitting the $\pm 2 \mu S$ error limit. Outside the measurement set period, the 3GPP timebase synchronisation mechanism alone should apply. The timebase error, due primarily to phase noise, will be random and unbiased during measurement period. Timebase drift, due to the small maximum frequency error of $0.1 \mathrm{ppm}$ [5], would be negligible over the duration of the intersample period. 
The Enhanced Timing Advance (ETA) method in summary, involves averaging the unbiased dithered timing advance measurement set over an appropriate number of samples using a tightly controlled MS timebase with a mean error of zero. The minimum duration of a measurement set of 11 TA samples for example, equates to 10 inter-burst periods i.e. $10 * 46.15 \mathrm{~ms}<0.5 \mathrm{sec}$. The method is exemplified below.

\section{Example:}

This example demonstrates the ETA principle without, for the sake of clarity, taking timebase synchronisation error into account.

Assume that the True Timing Advance (TTA) value is 10.35 symbol periods. TA in GSM is represented by integer values $(0-63)$, so in this case, TA would be set to 10. The application of linear dither to the measurement loop will result in TA values ranging from 10 to 11 as shown in Fig. 1 and Table 1.

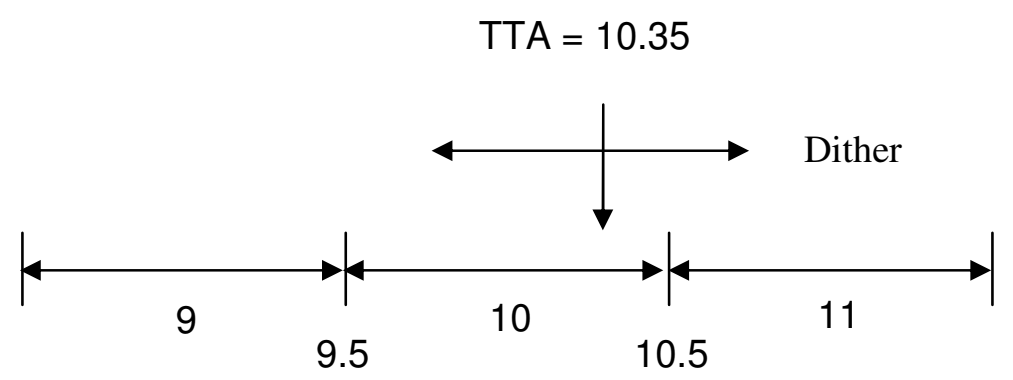

Timing Advance

Figure 1: Dither Mapping

where

$E T A=\sum$ SYMBOL PERIOD / N 
from table 1

$\mathrm{ETA}=114 / 11=10.36$

In practice, a 5 to 10 times improvement in accuracy and resolution should be achievable (depending on the number of samples in the measurement set), improving TA accuracy from 550m to considerably less than 100m (the FCC E-911 requirement).

\section{Conclusion:}

The letter firstly introduced a dither based measurement method to enhance measurement resolution. It then proposed a means by which to reduce the MS timebase error over the enhanced TA measurement period.

This letter has therefore presented a new method for MS location finding. This method would achieve enhanced TA resolution and accuracy with the potential to satisfy the FCC mobile E-911 accuracy requirement.

The method could in principle be implemented with only software changes. Only a minor increase in MS power consumption, as a result of increased $\mathrm{SCH}$ correlator usage, would result during the measurement period.

\section{References}

1 DEBLAUWE, N.: 'GSM Based Positioning: Techniques and Applications', pp. 7475, ISBN: 9789054874942

2 CONSTABILE, J.: 'Wireless Position Location Tutorial', Virginia Tech Wireless Symposium, June 4, 2010

3 Federal Communications Commission, Washington, FCC 07-108, "Wireless E911 Location Accuracy Requirements, Section 2 
4 3GPP TS 45.010: "Radio Subsystem Synchronization”, Para 6.2

5 3GPP TS 45.010: "Radio Subsystem Synchronization”, Para 6.1

\section{Authors' affiliations:}

R.S.C. Whitlock and T.W.C. Brown (Centre for Communication Systems Research, University of Surrey, Guildford, Surrey, GU2 7XH, United Kingdom)

E-mail: $\underline{\text { r.s.c.whitlock@gmail.com }}$

Table 1: Symbol Period Calculation of Dithered Transmission or Dithered Timing Window

\begin{tabular}{|c|c|c|c|}
\hline $\begin{array}{c}\text { TA MEASUREMENT } \\
\text { NUMBER }\end{array}$ & DITHER & TTA + DITHER & SYMBOL PERIOD \\
\hline 1 & -0.5 & 9.85 & 10 \\
\hline 2 & -0.4 & 9.95 & 10 \\
\hline 3 & -0.3 & 10.05 & 10 \\
\hline 4 & -0.2 & 10.15 & 10 \\
\hline 5 & -0.1 & 10.25 & 10 \\
\hline 6 & 0 & 10.35 & 10 \\
\hline 7 & 0.1 & 10.45 & 11 \\
\hline 8 & 0.2 & 10.55 & 11 \\
\hline 9 & 0.3 & 10.65 & 11 \\
\hline 10 & 0.4 & 10.75 & 11 \\
\hline 11 & 0.5 & 10.85 & \\
\hline
\end{tabular}

